\title{
Binary Gas Analyzer Based on a Single Gold Nanoparticle Photothermal Response
}

\author{
Xiangxiong Li, Jiani Hong, and Luning Zhang*
}

Cite This: ACS Omega 2020, 5, 27164-27170

Read Online

ABSTRACT: Although thermal conductivity gas analyzers are ubiquitous in industry, shrinking the sensing unit to a microscopic scale is rarely achieved. Since heat transfer between a metal nanoparticle and its ambient gas changes the temperature, refractive index, and density of the gaseous surrounding, one may tackle the problem using a single nanoparticle's photothermal effect. Upon heating by a $532 \mathrm{~nm}$ laser, a single gold nanoparticle transfers heat to the surrounding gas environment, which results in a change in the photothermal polarization of a $633 \mathrm{~nm}$ probe laser. The amplitude of the photothermal signal correlates directly with the concentration of binary gas mixture. In $\mathrm{He} / \mathrm{Ar}, \mathrm{He} / \mathrm{N}_{2}, \mathrm{He} / \mathrm{air}$, and $\mathrm{H}_{2} / \mathrm{Ar}$ binary gas mixtures, the signal is linearly proportional to the $\mathrm{He}$ and $\mathrm{H}_{2}$ molar concentrations up to about $10 \%$. The
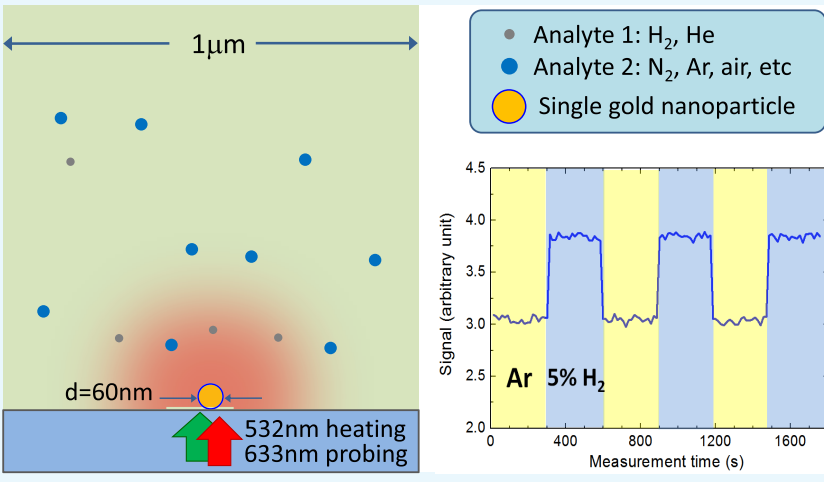
photothermal response comes from the microscopic gaseous environment of a single gold nanoparticle, extending from the nanoparticle roughly to the length of the gas molecule's mean free path. This study points to a way of sensing binary gas composition in a microscopic volume using a single metal nanoparticle.

\section{INTRODUCTION}

Gas sensors are becoming ubiquitous in our lives, with applications in the chemical industry, household safety, environmental protection, and border security, just to name a few. Several mechanisms for gas sensing have been studied for many years such as chromatography, thermal conductivity gas sensors, electrochemical gas sensors, semiconductor gas sensors, and optical absorption sensors. ${ }^{1-5}$ Optical sensors have a number of advantages in that they pose no risk of electric sparks while working ${ }^{6}$ and are capable of remote and nonintrusive monitoring along with a prompt optical response. Recently, a new family of optical gas sensoring based on the localized surface plasmon resonance (LSPR) of metal nanoparticles has started to attract attention. ${ }^{7-9}$ Using metal nanoparticles for sensing is a good approach to sensor miniaturization. ${ }^{10-12}$ Intrinsically, many metal nanoparticles exhibit environment-dependent LSPR, ${ }^{13-15}$ which not only reveals the physical and chemical changes of the environment but also shows how a nanoparticle interacts with its microscopic surroundings.

Reactions of gases with metal nanoparticles change the LSPR readily. Langhammer and co-workers ${ }^{16}$ demonstrated hydrogen sensing by LSPR spectroscopy using Pd nanodisks as a sensing platform. They showed that the adsorption and desorption of hydrogen on Pd nanoparticles could be measured accurately. ${ }^{17}$ Different types of Pd nanoparticles have since then been studied for hydrogen sensing such as Pd nanorings, ${ }^{18} \mathrm{Pd}$ nanocubes, ${ }^{19}$ and nanoscale Pd sandwich structures. ${ }^{20}$ In addition, a Pd nanoparticle could couple with a gold antenna to enhance its hydrogen sensitivity by concentrating an electric field in the coupling region. ${ }^{21}$ Gas sensing based on Ag nanoparticles was also demonstrated, by measuring the disappearance and reappearance of the LSPR peaks upon exposure to oxygen and hydrogen, respectively, after surface redox reactions. ${ }^{22}$

For nonreaction gas sensing, measuring the peak shift of LSPR induced by a refractive index change is possible. However, the extinction spectrum of a single metal nanoparticle usually exhibits a small signal-to-noise ratio (SNR) and a small peak shift upon tiny refractive index changes. For example, mixing $1 \% \mathrm{H}_{2}$ (or $1 \% \mathrm{He}$ ) in $\mathrm{Ar}$ at $100 \mathrm{kPa}$ induces about $2 \times 10^{-6}$ refractive index unit (RIU) change, a value almost impossible to measure by an LSPR peak shift. One may use an ensemble of nanoparticles to increase the SNR or use different sensing mechanisms. An array of silver and gold nanoparticles (AuNPs) has been fabricated for sensing $3 \times$ $10^{-4}$ RIU change in gases by an LSPR peak shift. ${ }^{23}$ The relatively good SNR was due to a large number of metal

Received: June 29, 2020

Accepted: September 9, 2020

Published: October 14, 2020 

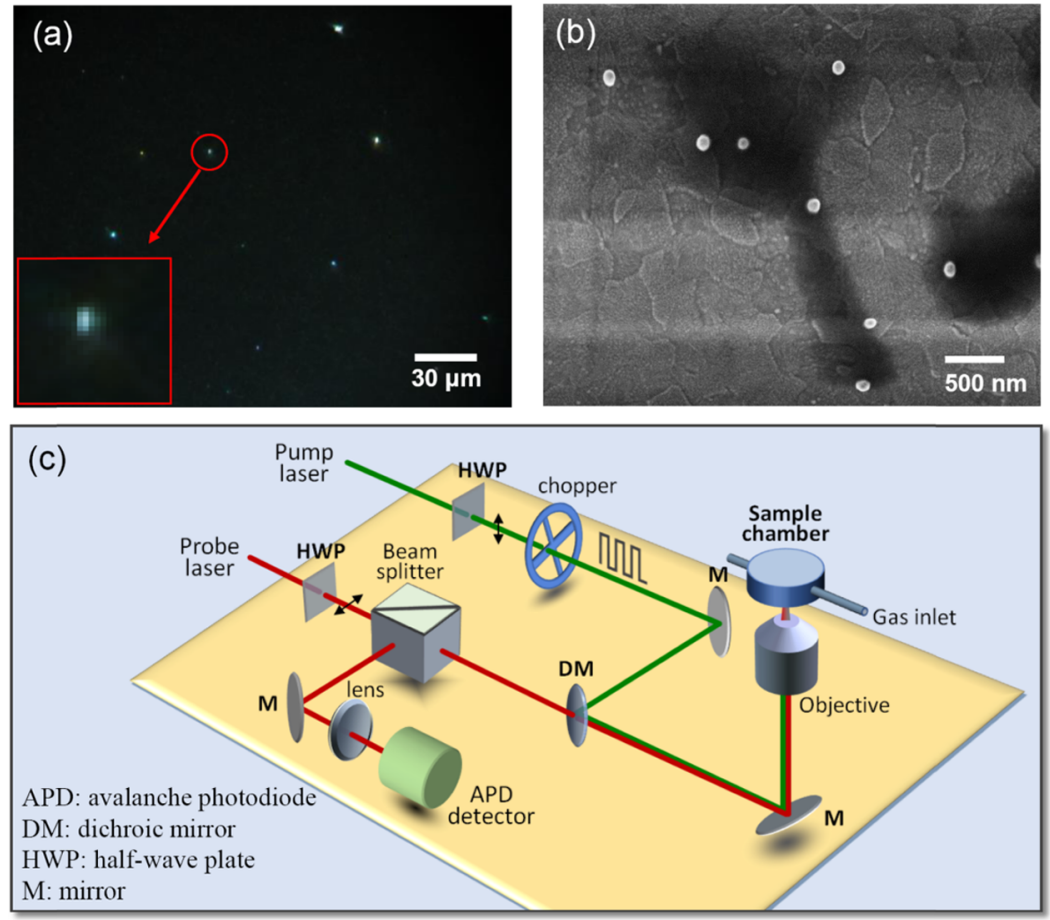

Figure 1. (a) Dark-field image of gold nanoparticles on a sapphire substrate in a sample. Green color in the image indicates an individual nanoparticle with resonance near $530 \mathrm{~nm}$. The inset shows an enlarged color spot. (b) Scanning electron microscopy image of gold nanoparticles used in our sample. Indium tin oxide is used for this imaging. (c) Schematic diagram of the experimental setup showing the pump (heating beam, 5-50 mW output, $(0.4-4.0) \times 10^{4} \mathrm{~W} / \mathrm{cm}^{2}$ on the sample) and probe $\left(21 \mathrm{~mW}\right.$ output, $4.7 \times 10^{3} \mathrm{~W} / \mathrm{cm}^{2}$ on the sample) laser beams leading to the sample chamber on an inverted microscope. The retroreflected probe beam passes through a beam splitter and enters the detector for lock-in amplification. The substrate with the AuNP serves as the bottom window of the chamber. An upright microscope with dark-field imaging above the chamber is not drawn.

nanoparticles in the test region. As demonstrated previously, the photothermal method is a noninvasive, highly sensitive, and real-time method, ${ }^{24-26}$ and is capable of nanoparticle chemical environment sensing. ${ }^{27-31}$ Using the photothermal response, one may circumvent the measurement of the LSPR spectrum and also obtain information of a nanoparticle's environment. Previous studies showed that photothermal imaging signals are sensitive to single protein binding events on metal nanoparticles. ${ }^{32}$ Such high sensitivity suggests potential use for gas sensing. Measuring gases using a single nanoparticle's photothermal response is rare. In theory, one may reason that heating an AuNP by a laser in resonance with its LSPR can subsequently heat up the surrounding gas, forming a local region of thermo-induced refractive index change. Consequently, an off-resonance probe laser may pick up the local refractive index variation in the gas. The photothermal signal is thus related to the gas thermal transport property.

This paper shows that a single AuNP's photothermal response can indeed be used as a gas analyzer. At small molar concentrations, the observed photothermal signal increases linearly with increasing $\mathrm{He}$ and $\mathrm{H}_{2}$ concentrations in $\mathrm{He} / \mathrm{Ar}, \mathrm{He} / \mathrm{N}_{2}, \mathrm{He}$ /air, and $\mathrm{H}_{2} / \mathrm{Ar}$ binary gas mixtures. In our photothermal method, it is not necessary to measure the LSPR spectral peak shift of a single AuNP. Instead, the photothermal response of the whole microscopic system, including the nanoparticle and the gas environment, is correlated with the gas composition.

\section{RESULTS AND DISCUSSION}

The dark-field image in Figure 1a shows a sample with greenish spots. These are individual gold nanoparticles having an LSPR close to $530 \mathrm{~nm}$. The scanning electron microscopy image of a typical sample is given in Figure $1 \mathrm{~b}$. The nanoparticles are reasonably uniform in size and mostly monodisperse on the substrate. The photothermal gas sensing setup is depicted in Figure 1c. It consists of an inverted microscope system for the heating $(532 \mathrm{~nm})$ and probe laser $(633 \mathrm{~nm})$ beams and an upright microscope (omitted in the figure) for sample viewing and dark-field imaging. Before each test, the focal spot of a laser beam is located and adjusted onto a single AuNP by viewing on the upright microscope. In principle, the morphology of the AuNP may change when illuminated by a focused laser beam. ${ }^{33}$ The shape changes in the AuNP are affected by the melting laser intensity as well as the electric field and surface diffusion coefficient of gold. The heating laser in our study is modulated in an on-off cycle, and the laser irradiance is much smaller compared to that reported by Kuhlicke et al. ${ }^{33}$ We may assume that our AuNPs are not melted. The operational condition of a single AuNP is shown to be stable for more than $30 \mathrm{~min}$ (see Figure S1 and SI section 1).

The photothermal response of a single AuNP in pure He gas exhibits good SNR, as is shown in Figure 2a. This figure compares a set of signals from two laser focal positions. The first one is a single AuNP, and the second is a blank region on the substrate, which gives the background. The background noise barely changes from vacuum up to $80 \mathrm{kPa}$ of $\mathrm{He}$. In pure $\mathrm{He}$, the AuNP's photothermal signal increases with pressure up 

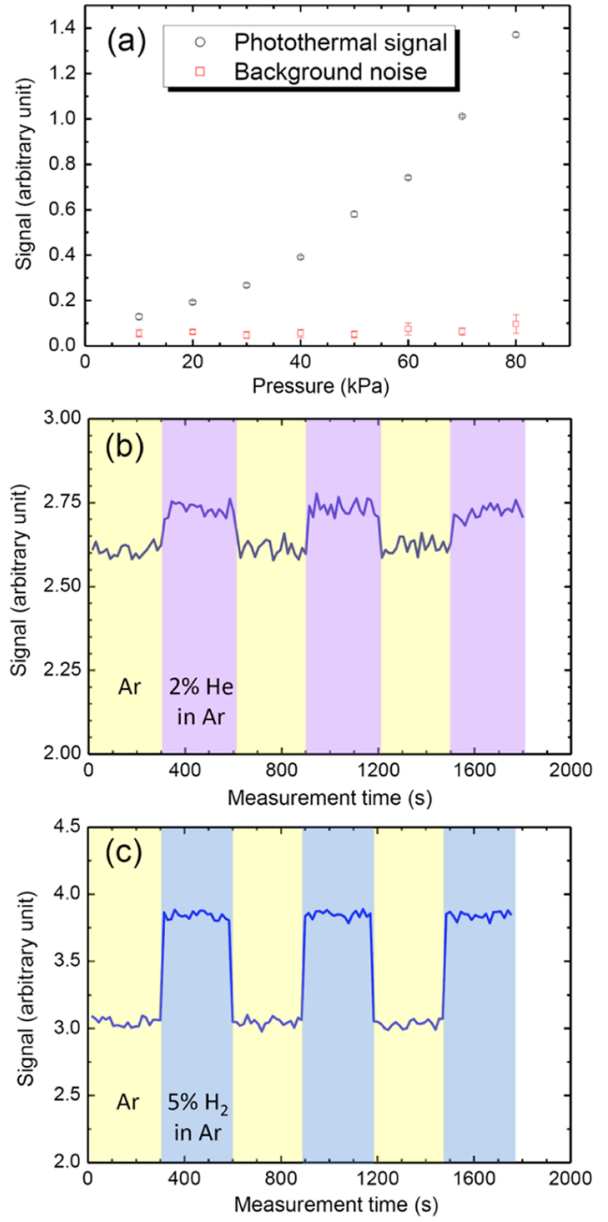

Figure 2. (a) Comparison of photothermal signals from the blank substrate without a nanoparticle and a gold nanoparticle. Both measurements are in He gas. (b) Change of the photothermal signal of a single gold nanoparticle as the ambient gas switches between $\mathrm{Ar}$ and $2 \% \mathrm{He}$ in Ar. (c) Change of the photothermal signal of a single gold nanoparticle as its ambient gas switches between $\mathrm{Ar}$ and $5 \% \mathrm{H}_{2}$ in Ar. The total pressure in (b) and (c) is $50 \mathrm{kPa}$.

to $80 \mathrm{kPa}$. The signal is a monotonic quadratic function of pressure $p$. Previous studies have shown that the photothermal signal $S$ has the property $S \propto n(\partial n / \partial T)^{25}$ and the refractive index $n$ of a gas has the relation $(n-1) \propto p / T .{ }^{34}$ Hence, $S$ is proportional to $p^{2}$, as shown in Figure S2.

The above results suggest that at a certain pressure, collisions between gas molecules and heated AuNPs increase the kinetic energy of the gas molecules. This leads to an increase in the temperature of the system, followed by an increase in the thermal conductivity of the gas. Thus, a photothermal signal arises when gas molecules are present. Obviously, if there is no AuNP, there is no photothermal signal since the probe beam is far from the LSPR peak. In an imaginative extreme case, we may assume that all gas molecules become stationary and there is no kinetic energy exchange. Even if the heating laser and probe laser enter the system, there is no photothermal signal whatsoever. In this case, even if the heating laser still heats up the AuNP, the probe laser simply does not "see" any change in the gaseous part of the whole microscopic system. Because the $633 \mathrm{~nm}$ probe laser is far from the LSPR peak of the AuNP, a heated nanoparticle without the surrounding gas cannot affect the probe beam. In other words, our photothermal signal is a direct probe of the microscopic gas environment.

Now, we move on to binary gas sensing using $\mathrm{He}$ in $\mathrm{Ar}$ and $\mathrm{H}_{2}$ in $\mathrm{Ar}$ as model systems. Like many commercial gas analyzers that are based on thermal responses, our photothermal sensing method does not have molecular selectivity. Therefore, we just experiment on gas mixtures with known chemical species but unknown concentrations. It is shown in Figure $2 \mathrm{~b}$ that when the chamber is filled alternately with pure $\mathrm{Ar}$ and $\mathrm{He}(2 \%)$ in $\mathrm{Ar}$, the photothermal signal follows the variation. The signal increases from $2.60 \pm 0.04$ for pure Ar to $2.73 \pm 0.04$ for $\mathrm{He}(2 \%) / \mathrm{Ar}$. In the case of $\mathrm{H}_{2}$, the signal is $3.00+0.04$ for pure $\mathrm{Ar}$ and increases to $3.80+0.04$ for $\mathrm{H}_{2}$ $(5 \%) / A r$. This is shown in Figure 2c. Since the two samples are different in Figure $2 b, c$, there is a difference in the absolute value. In terms of percentage change in the signal, $1 \% \mathrm{He}$ in $\mathrm{Ar}$ induces a signal increase of about $3 \%$, and $1 \% \mathrm{H}_{2}$ in $\mathrm{Ar}$ induces a signal increase of about $5 \%$.

Why does a small amount of $\mathrm{He}$ or $\mathrm{H}_{2}$ in Ar greatly change the photothermal signal? A look at the thermal conductivity $(\kappa)$ shows that Ar has a $\kappa$ of $17.7 \mathrm{~mW} \mathrm{~m}^{-1} \mathrm{~K}^{-1}$, much smaller than that of $\mathrm{He}\left(155.7 \mathrm{~mW} \mathrm{~m}^{-1} \mathrm{~K}^{-1}\right)$ or $\mathrm{H}_{2}\left(186.6 \mathrm{~mW} \mathrm{~m}^{-1}\right.$ $\mathrm{K}^{-1}$ ). Therefore, adding $\mathrm{He}\left(\right.$ or $\left.\mathrm{H}_{2}\right)$ in Ar would cause a rise in the $\kappa$ of the mixture, resulting in more efficient heat transfer between the heated AuNP and the gas species. An increase in the photothermal signal is then detected. As mentioned in the Introduction section, $1 \% \mathrm{He}$ or $\mathrm{H}_{2}$ in Ar causes about $2 \times 10^{-6}$ RIU change. Previously, sensors based on optical fiber MachZehnder interferometers were able to detect about $1 \times 10^{-5}$ RIU change. ${ }^{35}$ In our case, we do not directly measure the refractive index change of the system but rely on the thermal response. Thus, our photothermal gas sensing is not limited by the limit of detection (LOD) based on the RIU change.

It is possible to do quantitative analysis using the photothermal signal, which correlates with the gas molar concentration. Analyses of four gas mixtures are demonstrated: $\mathrm{He} / \mathrm{Ar}, \mathrm{He} / \mathrm{Air}, \mathrm{He} / \mathrm{N}_{2}$, and $\mathrm{H}_{2} /$ Ar. All tests are done at a fixed total pressure of $50 \mathrm{kPa}$. For each gas mixture, at least three tests are done to ensure reproducibility. When $\mathrm{He}$ is used as the guest species, we observe a linear response of signal versus $\mathrm{He}$ molar concentration up to $15 \%$ in all three host gases. In Figure 3a, the $y$-axis is called the signal increase level. It is calculated by taking the ratio of signal increase to the original signal of the pure host gas: $\left(S_{\text {guest gas }}-S\right) / S$. Three linear fitting results are shown in Figure 3a. Slopes differ in different host gases: $\mathrm{He} / \mathrm{Ar}$ is the largest and $\mathrm{He} / \mathrm{N}_{2}$ is the smallest. It is postulated that the photothermal signal increases following the increase in thermal conductivity of the medium. ${ }^{25,30,31}$ The validity of this assumption is shown by plotting the percentage increase of the calculated thermal conductivity (and also diffusivity) as a function of gas molar concentration (refer to Figure S3). Such plots efficiently reproduce the experimental trend quantitatively: $\mathrm{He} / \mathrm{Ar}$ has the largest slope and $\mathrm{He} / \mathrm{Air}$ and $\mathrm{He} / \mathrm{N}_{2}$ mixtures have smaller but similar slopes.

Hydrogen is a colorless, odorless, and highly flammable species that is widely used in the petroleum and chemical industries. The degree of safety at every stage of hydrogen utilization, including sensing, remains a significant challenge. $^{36,37}$ For demonstration, we show the sensing of $\mathrm{H}_{2}$ (from 0 to 5\%) in Ar in Figure 3b. A linear response is seen as the $\mathrm{H}_{2}$ molar concentration increases to $5 \%$, at a total pressure of $50 \mathrm{kPa}$. From the linear fitting and the error bar 

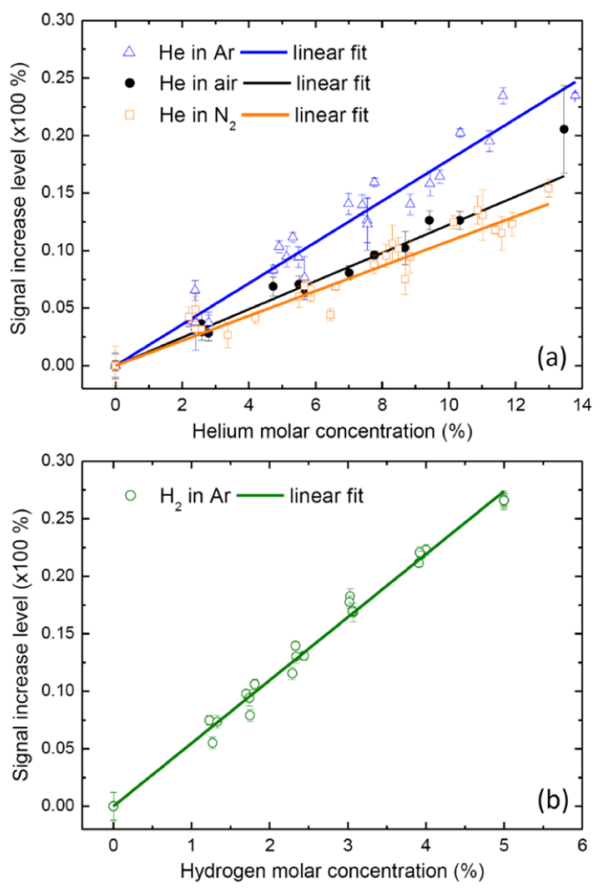

Figure 3. (a) Percentage of photothermal signal increase as a function of $\mathrm{He}$ concentration in $\mathrm{He} / \mathrm{Ar}, \mathrm{He} / \mathrm{Air}$, and $\mathrm{He} / \mathrm{N}_{2}$ mixtures. (b) Percentage of photothermal signal increase as a function of $\mathrm{H}_{2}$ concentration in a $\mathrm{H}_{2} / \mathrm{Ar}$ mixture. The total pressure of all measurements is $50 \mathrm{kPa}$. Slopes of linear fitting: $\mathrm{He} / \mathrm{Ar} 0.018, \mathrm{He} /$ Air $0.012, \mathrm{He} / \mathrm{N}_{2} 0.011$, and $\mathrm{H}_{2} / \mathrm{Ar} 0.055$.

measurements, we derive the LOD for $\mathrm{H}_{2}$ sensing to be $0.5 \%$ in Ar. If we were to measure the RIU change, it corresponds to about $10^{-6}$ RIU change. Detection of RIU change of such a small value is very difficult, yet the photothermal signal is capable of sensing it with a reasonable SNR, by alternatively measuring the heat transfer property and its effect on the probe beam.
For $\mathrm{H}_{2}$, the sensitivity of the photothermal signal is larger than that of $\mathrm{He}$. The signal level increases by about $27 \%$ as $\mathrm{H}_{2}$ increases from 0 to $5 \%$ in Ar. This increase is much larger than the mixtures containing $5 \% \mathrm{He}$ (signal increases by about 9,6 , and $5 \%$ for $\mathrm{He} / \mathrm{Ar}, \mathrm{He} / \mathrm{Air}$, and $\mathrm{He} / \mathrm{N}_{2}$, respectively). In Figure S4, we show plots of the percentage increase of thermal conductivity and thermal diffusivity for $\mathrm{H}_{2} / \mathrm{Ar}$ mixtures. The calculation has a slope that is about $50 \%$ higher than the experimental curve even though the trend is similar. This suggests that one cannot fully attribute the photothermal signal increase with added $\mathrm{H}_{2}$ to the thermal conductivity change. Other mechanisms such as convection near the AuNP, deviation of the gas mixture from ideal gas, and surface accommodation might contribute at the microscopic level. Nevertheless, the linear relationship of the photothermal signal with the analyte molar concentration indicates that a binary gas analyzer based on a single AuNP is possible.

Figure 4 illustrates the principles of the binary gas sensing. We may assume that the probe region is a hemispherical region having a diameter of approximately $600 \mathrm{~nm}$. This corresponds to the diffraction-limited focal point of a probe laser at $633 \mathrm{~nm}$. At $300 \mathrm{~K}$, the mean free path of gas species is about $270 \mathrm{~nm}$, just inside the laser's probe region (Figure 4a). When molecules collide with the heated AuNP surface and obtain more kinetic energy, such processes induce perceivable changes in the probe region. The frequency of molecular collision increases in the hot region. There is also a certain amount of mass transport from the hot region to the outer colder gas. Such outward molecular motion will encounter many collisions. The photothermal signal probably extends to a distance on a par with the mean free path, at the most a bit larger than that value. Further away, the probe laser will not be able to see a significant change within a small volume. If the increased motion extends to $3000 \mathrm{~nm}$ away, the effect will be distributed in a volume of about 1000 times the original region, when it extends merely $300 \mathrm{~nm}$ away from the AuNP.
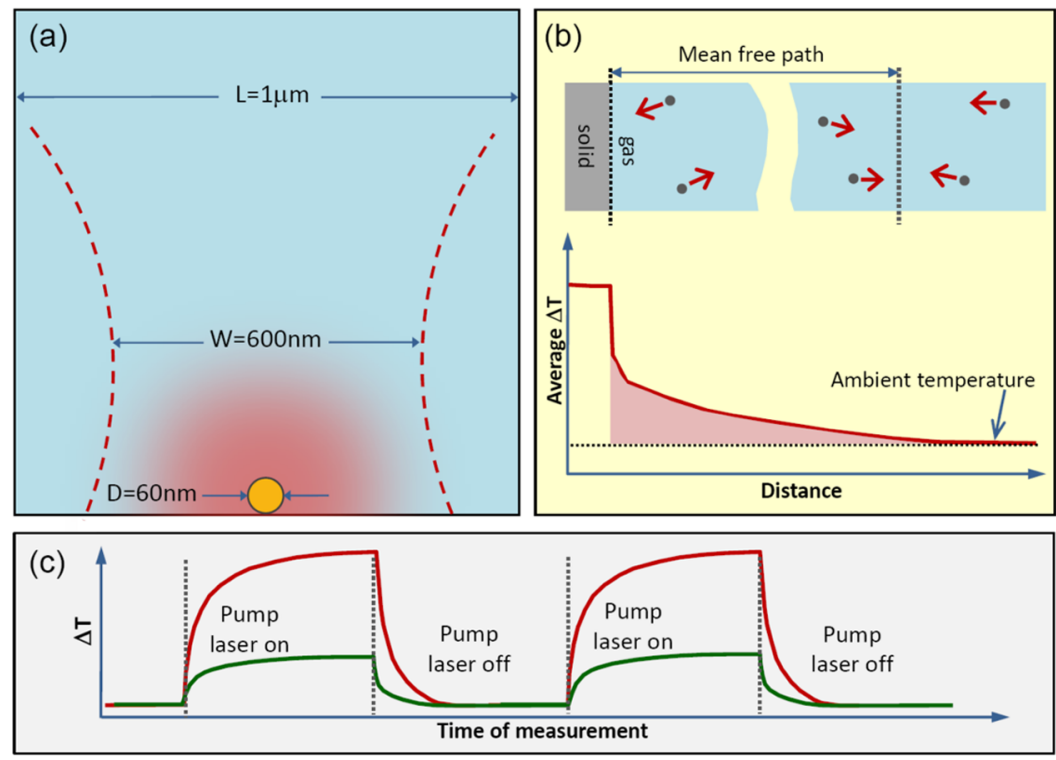

Figure 4. (a) Schematic diagram of the sample geometry in the laser focal spot region. Dotted lines represent the beam waist of a probe laser. (b) Illustration of the temperature profile at the interface of gold nanoparticles and ambient gas upon laser heating. The photothermal signal mainly comes from a region comparable to the mean free path. (c) Illustration of the temperature jump of gold nanoparticles (red line) and the surrounding gas (green line) during photothermal heating cycles. 
In Figure 4b, we show that the photothermal signal likely comes from a spherical region with radius on the order of the mean free path. This region has the largest kinetic energy exchange and the highest temperature rise. Based on this assumption, we estimate that the photothermal signal comes from a volume of about $10^{-19} \mathrm{~m}^{3}$ around a single AuNP. In this volume, there are about $2 \times 10^{6} \mathrm{Ar}$ and about $10^{4} \mathrm{H}_{2}$ molecules (assume LOD of $0.5 \% \mathrm{H}_{2}$ in Ar). Such estimations may be off by an order of magnitude, so it is probably safe to say that photothermal sensing is able to pick up signals from about $10^{4}-10^{5} \mathrm{H}_{2}$ molecules around a single AuNP. Figure $4 \mathrm{c}$ illustrates that within each photothermal cycle, the temperature of the AuNP first increases sharply and then the surrounding gas temperature follows. Within each cycle, the temperature should reache a semi-equilibrium value in the end. When the heating laser is switched off, the temperature of the nanoparticle drops suddenly. The gas temperature also drops. Both temperatures reach semi-equilibrium at the end of the cycle.

We can estimate the temperature rise at the interface between the AuNP and its gas environment using a simplified model reported recently. ${ }^{38}$ For steady-state with square-wave type of laser heating, the temperature of a nanoparticle can be regarded as reaching a steady value. In such a case, the energy absorbed by the nanoparticle should be dissipated through radiation loss and conduction to the gas environment. This can be written as

$$
E_{\text {abs }}=Q_{\text {radiative }}+Q_{\text {conduction }}
$$

This equation can be written in an explicit form to include experiment parameters. Equation 1 now becomes

$$
A k_{\mathrm{abs}} P=S\left[\sigma \varepsilon\left(T_{\mathrm{m}}^{4}-T_{0}^{4}\right)+\frac{G \kappa}{r_{0} G+\kappa}\left(T_{\mathrm{m}}-T_{0}\right)\right]
$$

where $A$ is the cross section of AuNP, $k_{\text {abs }}$ is the absorption coefficient, $P$ is the irradiance, $S$ is the surface area of the spherical AuNP, $\sigma$ is the Stefan-Boltzmann constant for radiation, $T_{\mathrm{m}}$ is the temperature of AuNP, $T_{0}$ is the original temperature, $\kappa$ is the thermal conductivity of the gas, $r_{0}$ is the AuNP radius, and $G$ is an adjustable parameter of thermal boundary conductance with a unit of $\mathrm{W} \mathrm{m} \mathrm{m}^{-2} \mathrm{~K}^{-1}$. When we put $r_{0}=30 \mathrm{~nm}, G=100 \mathrm{~W} \mathrm{~m}^{-2} \mathrm{~K}^{-1}$, and other experimental and literature parameters in eq 2 , the estimated $T_{\mathrm{m}}$ is approximately $1000 \mathrm{~K}$ at a medium pump irradiance of $10^{4}$ $\mathrm{W} / \mathrm{cm}^{2}$. Therefore, $\Delta T$ in the Figure $4 \mathrm{~b}$ plot is probably on the order of $700 \mathrm{~K}$. This is a large temperature rise for the AuNP, which might have been overestimated. However, since $G$ for sol id-gas heat transfer is several orders of magnitude smaller than that for the solid-liquid case, the temperature of the AuNP optically heated in gas should be much higher.

The diffusion of heat based on photothermal heating of nanostructures is a complex process, which is affected by nanoparticle radiation, ${ }^{38}$ size-dependent thermal conductivity, thermal boundary resistances, and LSPR. ${ }^{39}$ The heat transfer across the interface between a single, free-standing nanowire and surrounding air was quantitatively measured through laser thermography by Cheng and co-workers. ${ }^{40}$ The study quantified the heat transfer coefficient across the nanowireair interface over a wide range of pressures. Roder and coworkers developed a heat transport model to predict the heat diffusion of finite nanowires trapped by a single-beam laser trap. $^{41}$ The study demonstrated that the temperature distribution based on photothermal heating of nanowires is affected by numerous thermal, optical, and geometric parameters. They developed an analytical approach to predict the temperature distributions of one-dimensional structures, and elucidated the effect of size-dependent thermal parameters on nanoscale temperature distributions. ${ }^{42}$

Additionally, the ratio of the Stokes and anti-Stokes emission from resonantly excited gold nanoparticles has been used to measure the temperature. ${ }^{43,44}$ It has been demonstrated that the surface plasmon resonance could enhance radiative recombination of hot nanostructures. The effective temperature near a photothermal nanoparticle could also be estimated based on the amplitude and phase of the experimental signal. ${ }^{45}$ At the moment, we lack the spectral measurement capability to derive temperature using the Stokes and anti-Stokes emission. This will be the focus of our study in the near future.

In terms of the temporal and spatial resolution of our sensor, we have the following observations. The response of our AnNP-based analyzer is almost instantaneous. Considering the time required for the lock-in amplifier to reach an averaged value, the response time is on the order of a few seconds up changing the gas environment. This is similar to gas chromatography and other sensors based on thermal conductivity. Such a response time is enough for normal operation of hydrogen applications. Our photothermal imaging is spatially limited by the diffraction limit of our laser beams. For applications, when an increase in resolution is desired, one may use the nonlinear photothermal response ${ }^{46}$ or resort to a super-resolution imaging method. Suppose we can have a special probe laser that can be focused with a focal spot much smaller than $300 \mathrm{~nm}$, for example, near $50 \mathrm{~nm}$, we could use this probe laser to measure the location and distribution of the most prominent molecular heating area. We can find the temperature distribution near a single gold nanoparticle with sub-diffraction limit. This will enable us to plot experimental curves similar to Figure 4 b,c. Obviously, this is beyond the scope of this paper and may soon become feasible with the advancement of optics and development in nanoscopy imaging techniques.

\section{CONCLUSIONS}

By applying photothermal sensing on a single AuNP in different gas environments, we have shown that the photothermal signal is highly sensitive to the composition of a binary gas mixture. For principle demonstration, we have tested $\mathrm{He}$ / $\mathrm{Ar}, \mathrm{He} / \mathrm{Air}, \mathrm{He} / \mathrm{N}_{2}$, and $\mathrm{H}_{2} / \mathrm{Ar}$ binary mixtures. Individual AuNP's photothermal response is sensitive enough to determine the helium $(0-15 \%)$ or hydrogen $(0-5 \%)$ concentration. The LOD is about $1 \%$ for He and about $0.5 \%$ for $\mathrm{H}_{2}$. Previously, such small concentrations of guest molecules could induce only a $10^{-6}$ refractive index change, a value extremely difficult to detect. The success of photothermal gas sensing lies in two factors: first, we need not rely on the plasmonic energy response of a single gold nanoparticle by measuring the optical spectrum; second, we use the total thermal response of the gas environment plus the nanoparticle as a whole system. This paper illustrates that photothermal sensing might be useful for detecting gases such as hydrogen or helium within a microscopic region and in a noninvasive manner. 


\section{EXPERIMENTAL SECTION}

Materials. Our sample consists of $60 \mathrm{~nm}$ diameter AuNPs (BBI Solutions) deposited on a sapphire substrate that is precoated with a nanoporous $\mathrm{SiO}_{2}$ film using dip-coating. ${ }^{47}$ The nanoporous $\mathrm{SiO}_{2}$ film (with porosity $\sim 60 \%$ ) is a thermal isolation layer that reduces the heat loss to the substrate. An oxygen plasma cleaning procedure has been used on the $\mathrm{SiO}_{2}$ film prior to AuNP deposition. The increased hydrophilicity of the $\mathrm{SiO}_{2}$ film makes the spreading of AuNPs faster and results in a more uniform dispersion. In some tests, we also use a spincoated poly(methyl methacrylate) film. Such a coating between the AuNP and the substrate is known to decrease the heat transfer to the substrate ${ }^{25}$ and facilitate heat transfer to the gas environment. The substrate is assembled in a custom-built chamber, in which it serves as the bottom window for the laser beam path. The chamber also has a top sapphire window for three purposes: laser beam positioning, sample observation, and dark-field imaging of the AuNPs. After assembling, the chamber is filled with pure gas or binary mixtures with different compositions. During photothermal measurements, the pressure is fixed (typically $50 \mathrm{kPa}$ ) without further gas flow. Schematic diagrams of the gas distribution system and sample chamber are shown in Figure S5. After each composition switch, the gas is kept in the chamber for $300 \mathrm{~s}$ prior to the measurement. The AuNP exhibits strong absorption near $532 \mathrm{~nm}$ and weak absorption at $633 \mathrm{~nm}$ in a water suspension, as shown in Figure S6.

Instrument. The experimental setup is based on a homebuilt microscope equipped with an Olympus $100 \times$ objective $(\mathrm{NA}=0.8)$. An optical chopper (Thorlabs MC2000) is used to modulate the $532 \mathrm{~nm}$ heating laser (Cobolt, model Semba 5$50 \mathrm{~mW}$ output power, estimated irradiance $(0.4-4.0) \times 10^{4}$ $\mathrm{W} / \mathrm{cm}^{2}$ on the sample) at a desired frequency chosen between 500 and $900 \mathrm{~Hz}$. The continuous probe laser at $632.8 \mathrm{~nm}$ (Thorlabs HNL201L $21 \mathrm{~mW}$, estimated irradiance $4.7 \times 10^{3}$ $\mathrm{W} / \mathrm{cm}^{2}$ on the sample) retroreflects from the sample position and is focused on an avalanche photodiode (Thorlabs APD110A), which is connected to a lock-in amplifier (SRS model SR830) to pick up the periodic signal. The photothermal system characterization is shown in Figure S7, with data on the signal dependence on laser power and optical chopper modulation frequency.

\section{ASSOCIATED CONTENT}

\section{SI Supporting Information}

The Supporting Information is available free of charge at https://pubs.acs.org/doi/10.1021/acsomega.0c03124.

Figure S1: Plot of the photothermal signal as a function of time for stability verification; Figure S2: plot of photothermal signal as a function of gas pressure; Figure S3: plot of the percentage increase of thermal conductivity and thermal diffusivity for $\mathrm{He} / \mathrm{Ar}, \mathrm{He} / \mathrm{Air}$, and $\mathrm{He} / \mathrm{N}_{2}$, along with experimental data points; Figure S4: plot of the percentage increase of thermal conductivity and thermal diffusivity for $\mathrm{H}_{2} / \mathrm{Ar}$, along with experimental data points; Figure S5: schematic diagrams of the gas distribution system and sample chamber; Figure S6: UV-visible absorption spectrum of the AuNP suspension in water; and Figure S7: photothermal signal change as a function of laser power and optical chopper modulation frequency (PDF)

\section{AUTHOR INFORMATION}

\section{Corresponding Author}

Luning Zhang - School of Chemical Science and Engineering, Tongji University, Shanghai 200092, China; 이이.org/ 0000-0001-7088-0910; Email: luningzhang@tongji.edu.cn

\section{Authors}

Xiangxiong Li - School of Chemical Science and Engineering, Tongji University, Shanghai 200092, China

Jiani Hong - School of Chemical Science and Engineering, Tongji University, Shanghai 200092, China

Complete contact information is available at:

https://pubs.acs.org/10.1021/acsomega.0c03124

\section{Notes}

The authors declare no competing financial interest.

\section{ACKNOWLEDGMENTS}

This research is supported by the National Science Foundation of China, Project no. 21275107. This work is partially funded by (a) Fundamental Research Funds for the Central Universities (Tongji 1380219126, 22120180514) and (b) Shanghai Science and Technology Commission (14DZ2261100) through Shanghai Key Laboratory of Chemical Assessment and Sustainability.

\section{REFERENCES}

(1) Smith, D.; Spanel, P. The challenge of breath analysis for clinical diagnosis and therapeutic monitoring. Analyst 2007, 132, 390-396.

(2) Kohl, D. Oxidic Semiconductor Gas Sensors; Sberveglieri, G., Ed.; Springer: Berlin, 1992; pp 43-88.

(3) Bakker, E.; Qin, Y. Electrochemical sensors. Anal. Chem. 2006, $78,3965-3984$.

(4) Tierney, M.; Kim, J. Electrochemical gas sensor with extremely fast response times. Anal. Chem. 1993, 65, 3435-3440.

(5) Sakai, G.; Matsunaga, N.; Shimanoe, K.; Yamazoe, N. Theory of gas-diffusion controlled sensitivity for thin film semiconductor gas sensor. Sens. Actuators, B 2001, 80, 125-131.

(6) Wadell, C.; Syrenova, S.; Langhammer, C. Plasmonic hydrogen sensing with nanostructured metal hydrides. ACS Nano 2014, 8, 11925-11940.

(7) Allsop, T.; Arif, R.; Neal, R.; Kalli, K.; Kundrat, V.; Rozhin, A.; Culverhouse, P.; Webb, D. Photonic gas sensors exploiting directly the optical properties of hybrid carbon nanotube localized surface plasmon structures. Light: Sci. Appl. 2016, 5, No. e16036.

(8) Seo, D.; Park, G.; Song, H. Plasmonic monitoring of catalytic hydrogen generation by a single nanoparticle probe. J. Am. Chem. Soc. 2012, 134, 1221-1227.

(9) Della Gaspera, E.; Guglielmi, M.; Agnoli, S.; Granozzi, G.; Post, M. L.; Bello, V.; Mattei, G.; Martucci, A. Au nanoparticles in nanocrystalline $\mathrm{TiO} 2-\mathrm{NiO}$ films for SPR-based, selective $\mathrm{H} 2 \mathrm{~S}$ gas sensing. Chem. Mater. 2010, 22, 3407-3417.

(10) Lee, J. S.; Katoch, A.; Kim, J. H.; Sang, S. K. Effect of Au nanoparticle size on the gas-sensing performance of $\mathrm{p}-\mathrm{CuO}$ nanowires. Sens. Actuators, B 2016, 222, 307-314.

(11) Chen, R.; Morris, H. R.; Whitmore, P. M. Fast detection of hydrogen sulfide gas in the ppmv range with silver nanoparticle films at ambient conditions. Sens. Actuators, B 2013, 186, 431-438.

(12) Korotcenkov, G.; Brinzarib, V.; Gulina, L. B.; Cho, B. K. The influence of gold nanoparticles on the conductivity responseof $\mathrm{SnO} 2-$ based thin film gas sensors. Appl. Surf. Sci. 2015, 353, 793-803.

(13) Lu, X. M.; Rycenga, M.; Skrabalak, S. E.; Wiley, B.; Xia, Y. Chemical synthesis of novel plasmonic nanoparticles. Annu. Rev. Phys. Chem. 2009, 60, 167-192.

(14) Marlow, F.; Zwartkruis, F.; Malicki, J.; Neuhauss, S. C. F.; Abbas, L.; Weaver, M.; Driever, W.; Krezel, L. S. The Bakerian 
lecture: experimental relations of gold (and other metals) to light. Philos. Trans. R. Soc. London 1857, 147, 145-181.

(15) Kelly, K. L.; Coronado, E.; Zhao, L. L.; Schatz, G. C. The optical properties of metal nanoparticles: the influence of size, shape, and dielectric environment. J. Phys. Chem. B 2003, 107, 668-677.

(16) Langhammer, C.; Zoric, I.; Kasemo, B.; et al. Hydrogen storage in Pd nanodisks characterized with a novel nanoplasmonic sensing scheme. Nano Lett. 2007, 7, 3122-3127.

(17) Zoric, I.; Larsson, E. M.; Kasemo, B.; Langhammer, C. Localized surface plasmons shed light on nanoscale metal hydrides. Adv. Mater. 2010, 22, 4628-4633.

(18) Langhammer, C.; Larsson, E. M.; Zhdanov, V. P.; Zoric, I. Asymmetric hysteresis in nanoscopic single-metal hydrides: palladium nanorings. J. Phys. Chem. B 2012, 116, 21201-21207.

(19) Niu, W. X.; Zhang, W. Q.; Firdoz, S.; Lu, X. Controlled synthesis of palladium concave nanocubes with sub-10-nanometer edges and corners for tunable plasmonic property. Chem. Mater. 2014, $26,2180-2186$

(20) Strohfeldt, N.; Tittl, A.; Giessen, H. Long-term stability of capped and buffered palladium-nickel thin films and nanostructures for plasmonic hydrogen sensing applications. Opt. Mater. Express 2013, 3, 194-204.

(21) Liu, N.; Tang, M. L.; Hentschel, M.; Giessen, H.; Alivisatos, A. P. Nanoantenna-enhanced gas sensing in a single tailored nanofocus. Nat. Mater. 2011, 10, 631-6.

(22) Hu, J.; Wang, L.; Cai, W.; Li, W.; Zeng, H.; Zhao, L.; Liu, P. Smart and reversible surface plasmon resonance responses to various atmospheres for silver nanoparticles loaded in mesoporous $\mathrm{SiO}$. J. Phys. Chem. C 2009, 113, 19039-19045.

(23) Bingham, J. M.; Anker, J. N.; Kreno, L. E.; Van Duyne, R. P. Gas sensing with high-resolution localized surface plasmon resonance spectroscopy. J. Am. Chem. Soc. 2010, 132, 17358-9.

(24) Bialkowski, S. E. Photothermal Spectroscopy Methods for Chemical Analysis; John Wiley \& Sons: New York, 1996; pp 584.

(25) Gaiduk, A.; Yorulmaz, M.; Ruijgrok, P. V.; Orrit, M. Roomtemperature detection of a single molecule's absorption by photothermal contrast. Science 2010, 330, 353-356.

(26) Tokeshi, M.; Uchida, M.; Hibara, A.; Sawada, T.; Kitamori, T. Determination of subyoctomole amounts of nonfluorescent molecules using a thermal lens microscope: subsingle-molecule determination. Anal. Chem. 2001, 73, 2112-2116.

(27) Mawatari, K.; Kitamori, T.; Sawada, T. Individual detection of single-nanometer-sized particles in liquid by photothermal microscope. Anal. Chem. 1998, 70, 5037-5041.

(28) Gaiduk, A.; Ruijgrok, P. V.; Yorulmaz, M.; Orrit, M. Detection limits in photothermal microscopy. Chem. Sci. 2010, 1, 343-350.

(29) Selmke, M.; Marco, B.; Frank, C. Photothermal single-particle microscopy: detection of a nanolens. ACS Nano 2012, 6, 2741-2749.

(30) Cognet, L.; Stéphane, B.; Lasne, D.; Lounis, B. Photothermal methods for single nonluminescent nano-objects. Anal. Chem. 2008, $80,2288-2294$.

(31) Brusnichkin, A. V.; Nedosekin, D. A.; Proskurnin, M. A.; Zharov, V. P. Photothermal lens detection of gold nanoparticles: theory and experiments. Appl. Spectrosc. 2007, 61, 1191-1201.

(32) Zijlstra, P.; Paulo, P. M. R.; Orrit, M. Optical detection of single non-absorbing molecules using the surface plasmon resonance of a gold nanorod. Nat. Nanotechnol. 2012, 7, 379-382.

(33) Kuhlicke, A.; Schietinger, S.; Matyssek, C.; Busch, K.; Benson, $\mathrm{O}$. In situ observation of plasmon tuning in a single gold nanoparticle during controlled melting. Nano Lett. 2013, 13, 2041-2046.

(34) Clergent, Y.; Durou, C.; Laurens, M. Refractive index variations for argon, nitrogen, and carbon dioxide at $\lambda=632.8 \mathrm{~nm}$ (He-Ne Laser Light) in the range $288.15 \mathrm{~K} \leq \mathrm{T} \leq 323.15 \mathrm{~K}, 0<\mathrm{p}<110 \mathrm{kPa}$. J. Chem. Eng. Data 1999, 44, 197-199.

(35) Zhang, N. Y. M.; Li, K.; Zhang, N.; Zheng, Y.; Zhang, T.; Qi, M.; Shum, P.; Wei, L. Highly sensitive gas refractometers based on optical microfiber modal interferometers operating at dispersion turning point. Opt. Express 2018, 26, 29148-29158.
(36) Buttner, W. J.; Post, M. B.; Burgess, R.; Rivkin, C. An overview of hydrogen safety sensors and requirements. Int. J. Hydrogen Energy 2011, 36, 2462-2470.

(37) Chauhan, P. S.; Bhattacharya, S. Hydrogen gas sensing methods, materials, and approach to achieve parts per billion level detection: A review. Int. J. Hydrogen Energy 2019, 44, 26076-26099.

(38) Xi, Q.; Li, Y.; Zhou, J.; Li, B.; Li, J. Role of radiation in heat transfer from nanoparticles to gas media in photothermal measurements. Int. J. Mod. Phys. C 2019, 30, No. 1950024.

(39) Crane, M. J.; Zhou, X.; Davis, E. J.; Pauzauskie, P. J. Photothermal heating and cooling of nanostructures. Chem. Asian J. 2018, 13, 2575-2586.

(40) Cheng, C.; Fan, W.; Cao, J.; Ryu, S. G.; Ji, J.; Grigoropoulos, C. $\mathrm{P} . ; \mathrm{Wu}, \mathrm{J}$. Heat transfer across the interface between nanoscale solids and gas. ACS Nano 2011, 5, 10102-10107.

(41) Roder, P. B.; Smith, B. E.; Davis, E. J.; Pauzauskie, P. J. Photothermal heating of nanowires. J. Phys. Chem. C 2014, 118, $1407-1416$

(42) Roder, P. B.; Manandhar, S.; Devaraj, A.; Perea, D. E.; Davis, E. J.; Pauzauskie, P. J. Pulsed photothermal heating of one-dimensional nanostructures. J. Phys. Chem. C 2016, 120, 21730-21739.

(43) Carattino, A.; Caldarola, M.; Orrit, M. Gold nanoparticles as absolute nanothermometers. Nano Lett. 2018, 18, 874-880.

(44) Cai, Y. Y.; Sung, E.; Zhang, R.; Tauzin, L. J.; Liu, J. G.; Ostovar, B.; Zhang, Y.; Chang, W. S.; Nordlander, P.; Link, S. Anti-Stokes emission from hot carriers in gold nanorods. Nano Lett. 2019, 19, $1067-1073$

(45) Heber, A.; Selmke, M.; Cichos, F. Thermal diffusivity measured using a single plasmonic nanoparticle. Phys. Chem. Chem. Phys. 2015, 17, 20868-20872.

(46) Nedosekin, D. A.; Galanzha, E. I.; Dervishi, E.; Biris, A. S.; Zharov, V. P. Super-resolution nonlinear photothermal microscopy. Small 2014, 10, 135-142.

(47) Brinker, C. J.; Frye, G. C.; Hurd, A. J.; Ashley, C. S. Fundamentals of sol-gel dip coating. Thin Solid Films 1991, 201, $97-$ 108 